\title{
NUMERICAL INVESTIGATION OF CAVITATION IN MULTIDIMENSIONAL COMPRESSIBLE FLOWS*
}

\author{
KRISTEN J. DEVAULT ${ }^{\dagger}$, PIERRE A. GREMAUD ${ }^{\dagger}$, AND HELGE KRISTIAN JENSSEN J $^{\ddagger}$
}

\begin{abstract}
The compressible Navier-Stokes equations for an ideal polytropic gas are considered in $\mathbb{R}^{n}, n=2,3$. The question of possible vacuum formation, an open theoretical problem, is investigated numerically using highly accurate computational methods. The flow is assumed to be symmetric about the origin with a purely radial velocity field. The numerical results indicate that there are weak solutions to the Navier-Stokes system in two and three space dimensions, which display formation of vacuum when the initial data are discontinuous and sufficiently large. The initial density is constant, while the initial velocity field is symmetric, points radially away from the origin, and belongs to $H_{l o c}^{s}$ for all $s<n / 2$. In addition, in the one-dimensional case, the numerical solutions are in agreement with known theoretical results.
\end{abstract}

Key words. Navier-Stokes, Euler, compressible flow, cavitation, pseudospectral

AMS subject classifications. 35Q30, 65M70, 76N99

DOI. $10.1137 / 060652713$

1. Introduction. A long-standing open problem in the mathematical theory for fluid dynamics is the question of vacuum formation in compressible flow. Roughly stated, the issue is: Do there exist solutions of the Navier-Stokes system (3.1)-(3.3) for viscous compressible flow that exhibit vacuum (vanishing density) in finite time when the initial density is strictly bounded away from zero?

This problem is relevant from a modeling perspective as well as for theoretical results. The underlying assumption in the derivation of the Navier-Stokes equations from physical principles is that the fluid is nondilute and can be described as a continuum. A negative answer to the question above would thus provide self-consistency of the continuum assumption for the Navier-Stokes model. On the other hand, it is known that an a priori estimate of the form

$$
\text { for a constant } C=C(T): \quad C^{-1} \leq \rho(x, t) \leq C \quad \text { for all }(x, t) \in \mathbb{R}^{n} \times[0, T]
$$

(where $\rho$ denotes density) implies further estimates and would greatly facilitate existence proofs. For a discussion of this point, see Chapter 3 of [11].

Estimates of the above type are available for one-dimensional (1D) flow [26], even in the case of large and discontinuous data [15]. As reviewed in section 2, large efforts have been invested in searching for similar bounds in the multidimensional (multi-D) case. However, no such results seem to be currently known for the standard NavierStokes model with constant transport coefficients.

\footnotetext{
*Received by the editors February 22, 2006; accepted for publication (in revised form) April 27, 2007; published electronically September 26, 2007.

http://www.siam.org/journals/siap/67-6/65271.html

${ }^{\dagger}$ Department of Mathematics and Center for Research in Scientific Computation, North Carolina State University, Raleigh, NC 27695-8205 (kjdevaul@ncsu.edu, gremaud@ncsu.edu). The first author's research was partially supported by the National Science Foundation (NSF) through grant DMS-0410561. The second author's research was partially supported by the NSF through grants DMS-0244488 and DMS-0410561.

${ }^{\ddagger}$ Department of Mathematics, Penn State University, University Park, State College, PA 16802 (jenssen@math.psu.edu). This author's research was partially supported by the NSF through grants DMS-0206631 and DMS-0539549 (CAREER).
}

$$
1675
$$


To gain some insight, it is natural first to make a careful numerical study of the simplest possible scenario where one could expect cavitation in several space dimensions. This is the subject of the present work. In section 3, we consider 2D and 3D flows with symmetry for the full Navier-Stokes equations, as well as for the barotropic case (where pressure is assumed to be a function of density alone). The equations are written in a proper nondimensionalized form, and the problem is completed with appropriate initial and boundary conditions. More is known in the formal limit of infinitely large Reynolds numbers, i.e., in the case of inviscid fluids. The corresponding Euler equations and their solutions are used as a benchmark for the numerics. Various considerations regarding the inviscid case are discussed in section 4.

The above difficulties are mirrored on the numerical side in the form of various challenges regarding stability. The case of $1 \mathrm{D}$ flows illustrates this issue. Riemann data with large jumps may lead to vacuum formation for the Euler equations while, at least for Hoff's solutions [15], no vacuum occurs for solutions to the corresponding Navier-Stokes equations. Minimizing the amount of numerical diffusion is thus paramount. A splitting method is used between the convective part, corresponding to the Euler equations, and the diffusive part. The hyperbolic part is solved by computing local similarity solutions. Those solutions are then "diffused," and the process is repeated at the next time step. A highly accurate pseudospectral spatial discretization is used; see section 5. The stiffness [1] of the discretized-in-time system increases as the density $\rho$ goes to zero; for $\rho=0$, the system is infinitely stiff and has degenerated from being purely differential to being differential algebraic. Several numerical schemes, none of them explicit, are known to handle this kind of difficulty [1, 12] (at least for some problems presenting this type of structure). A backward difference formula (BDF)-type method is considered here. This approach has been successfully tested with respect to mass conservation and energy balance. Finally, a test over the magnitude of the density has to be done to determine whether "vacuum" has been reached, an arduous task in finite precision computations! The solution is analyzed both for its magnitude and its behavior in phase space.

The numerical experiments are set up so that vacuum, if any, appears first at a known point (the origin). The calculations do not attempt to track the solution past vacuum formation. The detection of the vacuum itself requires density to be less than $10^{-14}$ (see criterion (6.1)), i.e., just slightly above what corresponds to machine epsilon for the IEEE double precision machines used in the experiments.

Our detailed numerical study is described in section 6 . It indicates that vacuum formation indeed occurs for multi-D symmetric flows for sufficiently large and discontinuous initial data, i.e., in the regime of high Mach number and high Reynolds number. Section 7 contains a brief summary and a conclusion of our findings.

\section{Challenges and relation to other works.}

2.1. Theoretical issues. There is a voluminous literature on compressible flow. A short review of work relevant to vacuum formation and a priori estimates follows.

2.1.1. 1D flows. For 1D flow, i.e., multi-D flow with planar symmetry, much stronger results are known than what is currently available for higher dimensions. A seminal work of Kazhikhov and Shelukhin [26] considers the full 1D Navier-Stokes system for an ideal polytropic gas. Building on earlier work by Kanel [21] and Kazhikhov [25], the global existence and uniqueness of a smooth $\left(W^{1,2}\right)$ solution is proved in [26] for arbitrarily large and smooth data. In particular, a priori bounds of the type (1.1) are established. Similar results for more general gases can be found 
in [4], [5], [24].

Highly relevant to the present work is an extension to large and rough (possibly discontinuous) data established by Hoff [15]. This result pertains to isentropic or isothermal flow with data $\left(\rho_{0}, u_{0}\right)$ satisfying

$$
\rho_{0} \in L^{\infty}(\mathbb{R}), \quad \operatorname{essinf}_{\mathbb{R}} \rho_{0}>0,
$$

and

$$
\rho_{0}-\bar{\rho}, \quad u_{0}-\bar{u} \in L^{2}(\mathbb{R}),
$$

where $\bar{\rho}, \bar{u}$ are monotone functions that agree outside a bounded interval with the limiting values of $\rho$ and $u$ at $\pm \infty$. Hoff [15] proves that there exists a global weak solution which satisfies (1.1). This result shows that there is at least one solution of the Navier-Stokes equations that does not exhibit cavitation, even for Riemann-type data with arbitrarily large jumps. (For an extension of this result to certain flows for the full Navier-Stokes system see [20].) This is in contrast to the 1D inviscid Euler equations, for which it is well known that vacuum formation can occur [36]. The present numerical study does not contradict Hoff's result. Indeed, while our results clearly indicate the possibility of vacuum formation in higher dimensions, we have not numerically observed cavitation in solutions of the 1D Navier-Stokes system.

A significant result concerning cavitation in 1D flow is given by Hoff and Smoller [19]. They demonstrate that any, everywhere defined, weak solution of the NavierStokes (barotropic or full) system which satisfies some natural weak integrability assumptions cannot contain a vacuum in a nonempty open set unless the initial data do so. (For a refinement of this result, see [9].) Xin and Yuan [38] have recently performed a corresponding analysis for spherically symmetric solutions in $\mathbb{R}^{2}$ and $\mathbb{R}^{3}$. For everywhere defined solutions they give detailed information on the behavior of vacuum regions (if any), and they also provide sufficient conditions to rule out cavitation.

2.1.2. Multi-D flows. Much less is known about compressible flow in higher dimensions. Global existence of weak solutions is a formidable problem, and the theory is far from complete. Roughly speaking, currently known results are of two types: (A) for large and rough data that possibly contain vacuum states, and (B) for small, rough (discontinuous) data with $\operatorname{ess} \inf \rho_{0}>0$.

In the former case, Lions [28] has established existence of global weak solutions in the case of compressible barotropic flow. For recent extensions, including results for the full Navier-Stokes system, see [10], [11] and references therein. It is not known if a bound of the type (1.1) holds for these solutions when the data satisfy ess inf $\rho_{0}>0$.

More is known for "small" data, i.e., data close to a constant state in a suitable norm. In particular, small and sufficiently smooth data generate global smooth solutions without cavitation for the full Navier-Stokes system [31], [22], [23]. For a representative result in the case of barotropic flow, see Chapter 9 in [33]. In a series of papers [6], [7], [8], Danchin has established global existence, and also uniqueness, of compressible flows in several space dimensions for solutions in so-called critical spaces. For flows with even less regularity, possibly with discontinuities across hypersurfaces, Hoff [14] has shown that if the data are sufficiently close to a constant state, in a suitable norm, and with initial density and temperature bounded away from zero, then there exists a global weak solution with the same properties at all later times. No corresponding result seems to be known for large data in several space dimensions. 
There are somewhat stronger results available for the type of symmetric (quasi-1D) flows that we consider in this paper. For isothermal flow with spherical symmetry, Hoff [13] establishes existence of a global weak solution for large symmetric data. The solution is obtained as the limit of solutions in shells $\{0<a \leq r \leq b\}$ as $a \downarrow 0$. By rewriting the equations in Lagrangian coordinates and exploiting the energy estimate, certain a priori bounds are obtained that are independent of the inner radius a. However, while guaranteeing existence of a weak solution, the available a priori bounds do not seem strong enough to determine whether the constructed solution contains a vacuum at the center of motion. (For an extension of this result to the full Navier-Stokes system, see [17].)

We note that the higher the dimension of the space, the easier it should be to generate a vacuum, as the fluid is free to move in more directions. This can be quantified for the corresponding inviscid system. Consider the isentropic Euler equations with spherically symmetric Riemann-type data. Let the initial velocity field have constant magnitude $\bar{u}$ and be directed radially away from the origin. In this case, there is a threshold value $\hat{u}(n)$ of $\bar{u}$, depending on the dimension $n$, above which a vacuum is formed immediately [43]. One can verify that $\hat{u}(1)>\hat{u}(2)>\hat{u}(3)$. For the 1D Navier-Stokes system, we know from Hoff's result [13] that there exists a weak solution without vacuum; i.e., " $\hat{u}(1)=\infty$ " for these solutions. However, in higher dimensions it may well be that there are solutions with strictly positive density everywhere at time zero, but which develop a vacuum at later times. ${ }^{1}$ There are also technical reasons that seem to prevent a priori bounds on the density in higher dimensions. More precisely, in the 1D analysis of [26], [13] the bounds on the density are derived from the a priori bounds one gets "for free" from the equations themselves. These are integral bounds that are strictly stronger in one dimension than in higher dimensions due to the geometrical factor of $r^{n-1}$ in the space integrals (i.e., $d x=$ const. $r^{n-1} d r$ ).

\subsection{Additional remarks.}

Cavitation and uniqueness. The issue of cavitation is closely related to the question of uniqueness and to the concept of solution that one works with. To illustrate this consider the 1D Navier-Stokes equations with Riemann-type data:

$$
\rho_{0}(x) \equiv \bar{\rho}>0, \quad u_{0}(x)= \begin{cases}-\bar{u} & \text { for } x<0, \\ \bar{u} & \text { for } x>0,\end{cases}
$$

where $\bar{u}>0$. One weak solution to this problem is provided by Hoff's result [15], and this solution does not exhibit cavitation. However, a different solution can also be constructed, with the same data, by piecing together solutions of two disjoint flows into surrounding vacuum. More precisely, consider the two sets of initial data

$$
\rho_{0}^{-}(x)=\left\{\begin{array}{ll}
\bar{\rho} & \text { for } x<0, \\
0 & \text { for } x>0,
\end{array} \quad u_{0}^{-}(x)= \begin{cases}-\bar{u} & \text { for } x<0, \\
\emptyset & \text { for } x>0,\end{cases}\right.
$$

and

$$
\rho_{0}^{+}(x)=\left\{\begin{array}{ll}
0 & \text { for } x<0, \\
\bar{\rho} & \text { for } x>0,
\end{array} \quad u_{0}^{+}(x)= \begin{cases}\emptyset & \text { for } x<0 \\
\bar{u} & \text { for } x>0\end{cases}\right.
$$

(Here $\emptyset$ indicates that the velocity is left undefined where there is no matter.) One can now construct solutions $\left(\rho^{-}, u^{-}\right)$and $\left(\rho^{+}, u^{+}\right)$corresponding to these data, which

\footnotetext{
${ }^{1}$ Furthermore, the possibility remains that there are other weak solutions exhibiting vacuum even in one dimension (see below for further comments on uniqueness).
} 
in addition satisfy a physical no-traction boundary condition along a vacuum-fluid interface; see [4], [5], [24], [25]. By concatenating the two solutions, a solution to the original problem (2.1) is obtained, and in this solution an open vacuum region is present from time $t=0+$ (and staying at least for a short time). In the region between the two solutions, one may simply consider the flow to be undefined. Without going into the discussion of which solution is more relevant, we note that Hoff's solution [15] is defined everywhere on $\mathbb{R} \times \mathbb{R}_{+}$, while the second solution is defined only on the support of its density. The issue of nonuniqueness for 1D compressible Navier-Stokes in connection with vacuums is treated in detail by Hoff and Serre [18].

For flow in several space dimensions, the picture is less clear since we do not even know if there is a solution without cavitation in this case. The present work indicates that there is at least one solution where a vacuum forms. Uniqueness of general weak solutions, i.e., without any regularity assumptions beyond what is necessary to make sense of a weak formulation, is not known. On the other hand, sufficiently smooth and small solutions are unique (see [31]), as are flows belonging to critical spaces (see Danchin [6], [7], [8]). To the best of our knowledge, the only uniqueness result for flows with possible discontinuities in the density field is given in a recent work by Hoff [16].

Continuum assumption and physical boundary conditions. In view of the $1 \mathrm{D}$ examples above, one should be cautious in making claims about the "physicality" of constructed or computed solutions to the standard Navier-Stokes model (3.1)-(3.3) in the low-density regime. Of course, from a modeling point of view, this is not surprising. The Navier-Stokes system is derived under the assumption that the fluid can be described as a continuum with everywhere strictly positive mass density. Issues related to nonuniqueness in the presence of vacuum are therefore not surprising. For a discussion of this point, see [11]. It is also known [37] that the lifespan of smooth, everywhere defined solutions to the Navier-Stokes system (with vanishing heat conductivity) is finite whenever the initial density is compactly supported.

Once a vacuum has developed, one should impose the physical boundary condition of vanishing traction at the vacuum-fluid interface. In other words, the vacuum should not exert a force on the fluid. Note that in the present work we track the onset of vacuum formation, not its subsequent evolution.

Well-posedness. In view of both physical arguments as well as the apparent lack of good a priori estimates for the standard Navier-Stokes model, it is natural to ask whether more accurate models would lead to stronger results. In particular, models where the transport coefficients $\lambda, \mu, \kappa$ depend on the thermodynamical state have been considered. ${ }^{2}$ Recent results for such models are given in [2] and [32]. Several issues pertaining to well-posedness in the presence of vacuum have been analyzed in [29], [30], [39], [40], [41], [42]. These results show that the nonuniqueness observed in [18] can be attributed to the unphysical assumption of constant viscosity coefficient. The corresponding problem for the full system appears to require new methods. For results in this direction for the full $1 \mathrm{D}$ system, see [4], [5], [24], and see the recent monograph by Feireisl [11] for the multi-D case.

2.3. Precise formulation. After the above remarks, our original question can be formulated precisely as follows. Given the standard multidimensional NavierStokes model with constant transport coefficients, let the pressure be that of an ideal

\footnotetext{
${ }^{2}$ In the isentropic case a relevant assumption is that the viscosity $\mu$ depends on the density. In accordance with kinetic theory it is natural to consider the case with $\mu \sim \rho^{k}$, for a constant $k>0$.
} 
polytropic gas or, in the case of barotropic flow, of the form $A \rho^{\gamma}$ with $\gamma \geq 1$. Consider the initial-boundary value problem in a ball centered at the origin, with initial density strictly bounded away from zero and with a possibly discontinuous initial velocity field. Then: Does there exist an everywhere defined weak solution of the equations with the property that its density reaches zero in finite time?

3. The full compressible Navier-Stokes equations. Consider the compressible Navier-Stokes equations for a Newtonian fluid in $\mathbb{R}^{n}, n=1, n=2$, or $n=3$, with no external forces or heat sources. The invariant form of the equations in spatial (Eulerian) formulation is

$$
\begin{aligned}
\rho_{t}+\operatorname{div}(\rho \vec{u}) & =0, \\
(\rho \vec{u})_{t}+\operatorname{div}(\rho \vec{u} \otimes \vec{u}) & =\operatorname{grad}(-p+\lambda \operatorname{div} \vec{u})+\operatorname{div}(2 \mu D), \\
\mathcal{E}_{t}+\operatorname{div}((\mathcal{E}+p) \vec{u}) & =\operatorname{div}(\lambda(\operatorname{div} \vec{u}) \vec{u}+2 \mu D \cdot \vec{u}-\vec{q}),
\end{aligned}
$$

where $\rho$ is the density, $\vec{u}=\left(u_{1}, \ldots, u_{n}\right)^{T}$ is the fluid velocity, $p$ is the pressure, $\mathcal{E}$ is the total energy, $D$ is the deformation rate tensor, $\vec{q}$ is the heat flux vector, and $\lambda$ and $\mu$ are the viscosity coefficients. Equations (3.1)-(3.3) are often referred to as the continuity equation, the conservation of momentum equation, and the conservation of energy equation, respectively. We also have

$$
\mathcal{E}=\rho\left(e+|\vec{u}|^{2} / 2\right), \quad D_{i j}=\left(\partial_{i} u_{j}+\partial_{j} u_{i}\right) / 2, \quad \vec{q}=-\kappa \nabla \theta,
$$

where $e$ stands for the internal energy, $\kappa$ is the coefficient of heat conductivity, and $\theta$ is the temperature. In what follows, we restrict ourselves to the study of ideal and polytropic (perfect) gases such that

$$
p=\mathcal{R} \rho \theta, \quad e=c_{v} \theta,
$$

where $\mathcal{R}$ is the gas constant and $c_{v}$ is the specific heat at constant volume. The local sound speed $c$ is then given by

$$
c=\sqrt{\frac{\gamma p}{\rho}}
$$

where $\gamma=1+\mathcal{R} / c_{v}$ is the adiabatic exponent. All the transport coefficients $c_{v}, \lambda, \mu, \kappa$ are assumed to be constant. For the derivation of the equations, see, e.g., [33], [34].

3.1. Equations for symmetric flow. We next consider the case of flow with symmetry; i.e., the velocity is directed (radially when $n=2,3$ ) away from the origin, and all quantities are functions only of the distance to the origin and of time. Let $x$ denote a point in space, and set $r=|x|$. Setting

$$
\rho(r, t)=\rho(x, t), \quad \vec{u}(x, t)=u(r, t) \frac{x}{r}, \quad \text { etc. },
$$

leads to the following system of equations:

$$
\begin{aligned}
\rho_{t}+(\rho u)_{\xi} & =0, \\
\rho\left(u_{t}+u u_{r}\right)+p_{r} & =\nu u_{\xi r}, \\
c_{v} \rho\left(\theta_{t}+u \theta_{r}\right)+p u_{\xi} & =\kappa \theta_{r \xi}+\nu\left(u_{\xi}\right)^{2}-\frac{2 m \mu}{r^{m}}\left(r^{m-1} u^{2}\right)_{r},
\end{aligned}
$$

where we have used the notation

$$
m=n-1, \quad \partial_{\xi}=\partial_{r}+\frac{m}{r}, \quad \nu=\mu+2 \lambda .
$$

(Note that $\partial_{\xi r} \neq \partial_{r \xi}$ for $n=2$ and $n=3$.) 
We consider spherically symmetric flows (3.5)-(3.7) in the interior of the interval/ disk/ball $B_{b}$ of fixed outer radius $b$ :

$$
\rho(r, 0)=\rho_{0}(r), \quad u(r, 0)=u_{0}(r), \quad \theta(r, 0)=\theta_{0}(r) \quad \text { for } r \leq b .
$$

In the 1D case we require that (3.8) hold for all $|r| \leq b$. Throughout, we consider only the case where the gas is set in motion in the outward direction; i.e., we assume that $u_{0}$ is a nonnegative function in two and three dimensions and is odd with positive values for $r>0$ in one dimension. Suitable boundary conditions are discussed in 3.4.

3.2. Nondimensional form of symmetric equations. Using the initial data, characteristic length, velocity, density, and temperature can be defined as follows:

$$
\begin{aligned}
\bar{r} & :=b, \\
\bar{u} & :=\max _{0 \leq r \leq b}\left|u_{0}(r)\right|, \\
\bar{\rho} & :=\max _{0 \leq r \leq b} \rho_{0}(r), \\
\bar{\theta} & :=\max _{0 \leq r \leq b} \theta_{0}(r) .
\end{aligned}
$$

From these we define characteristic time and pressure by

$$
\begin{aligned}
\bar{t} & :=\frac{\bar{r}}{\bar{u}}, \\
\bar{p} & :=p(\bar{\rho}, \bar{\theta}) .
\end{aligned}
$$

The dimensionless independent variables are then

$$
R:=\frac{r}{\bar{r}}, \quad T:=\frac{t}{\bar{t}},
$$

and the dimensionless dependent variables are

$$
D:=\frac{\rho}{\bar{\rho}}, \quad U:=\frac{u}{\bar{u}}, \quad \Theta:=\frac{\theta}{\bar{\theta}}, \quad P:=\frac{p}{\bar{p}} .
$$

Regarding $D, U, \Theta, P$ as functions of $R$ and $T$, we obtain the nondimensionalized system

$$
\begin{aligned}
\rho_{t}+(\rho u)_{\xi}= & 0 \\
\rho\left(u_{t}+u u_{r}\right)+\frac{1}{\gamma M^{2}}(\rho \theta)_{r}= & \frac{1}{\operatorname{Re}} u_{\xi r}, \\
\rho\left(\theta_{t}+u \theta_{r}\right)+(\gamma-1) \rho \theta u_{\xi}= & \frac{1}{\operatorname{Pr} \operatorname{Re}} \theta_{r \xi} \\
& +\gamma(\gamma-1) \frac{\mathrm{M}^{2}}{\operatorname{Re}}\left(\left(u_{\xi}\right)^{2}-\frac{2 m \mu}{\nu} \frac{\left(r^{m-1} u^{2}\right)_{r}}{r^{m}}\right)
\end{aligned}
$$

where we have reverted to the original symbols and where

$$
\begin{aligned}
\mathrm{M} & :=\frac{|\bar{u}|}{\bar{c}}=\text { Mach number, } \quad \bar{c}=\text { sound speed }=\sqrt{\frac{\gamma \bar{p}}{\bar{\rho}}}, \\
\operatorname{Re} & :=\frac{\bar{r} \bar{\rho} \bar{u}}{\nu}=\text { Reynolds number, } \\
\operatorname{Pr} & :=\frac{\nu c_{v}}{\kappa}=\text { Prandtl number. }
\end{aligned}
$$

These equations are valid also for $n=1(m=0)$, provided that $r$ is interpreted as the position along the $x$-axis; in this case $\partial_{\xi}=\partial_{r}=\partial_{x}$. 
3.3. Barotropic equations. If the pressure $p$ is considered as a function of the density $\rho$ only, the energy equation (3.7) decouples from the mass and momentum conservation equations (3.5), (3.6). Here, we consider exclusively the isentropic and isothermal cases where

$$
p(\rho)=a \rho^{\gamma}, \quad \gamma \geq 1,
$$

and where $a>0$ is a constant. A nondimensional version of the barotropic equations can be derived in very much the same way as (3.9)-(3.11) were derived, except for the characteristic pressure, which is now taken as

$$
\bar{p}=p(\bar{\rho})=a \bar{\rho}^{\gamma} .
$$

This leads to the nondimensionalized system for barotropic flow

$$
\begin{aligned}
\rho_{t}+(\rho u)_{\xi} & =0, \\
\rho\left(u_{t}+u u_{r}\right)+\frac{1}{\gamma \mathrm{M}^{2}}\left(\rho^{\gamma}\right)_{r} & =\frac{1}{\operatorname{Re}} u_{\xi r},
\end{aligned}
$$

where the Mach number at the reference state is now given by

$$
M:=\frac{|\bar{u}|}{\bar{c}}=\frac{|\bar{u}|}{a \gamma \bar{\rho}^{\gamma-1}} .
$$

3.4. Initial and boundary conditions: Balance relations. The following initial and boundary conditions are considered throughout:

$$
\begin{aligned}
& \rho(r, 0)=\rho_{0}(r)=1 \quad \text { for } r \geq 0, \quad u(r, 0)=u_{0}(r)= \begin{cases}1 & \text { if } r>0 \\
0 & \text { if } r=0\end{cases} \\
& u(1, t)=1, \quad t>0,
\end{aligned}
$$

where $r=1$ corresponds now to the outer boundary of the computational domain. Furthermore, by symmetry, one has

$$
u(0, t)=0, \quad t>0 .
$$

A calculation shows that the initial velocity field is in $H_{l o c}^{s}\left(\mathbb{R}^{n}\right)$ for all $s<n / 2$.

It may seem more natural to consider a homogeneous condition of the type $u(1, t)=0$, instead of (3.16), since existence of weak solutions has been established in the former case. However, such a condition leads to steep gradients and unwanted numerical boundary layer effects on the outer boundary of the domain. A vanishing boundary condition at $x=1$ would thus complicate the numerical resolution of the problem by making it more susceptible to spurious oscillations. In any case, under the above type of initial conditions (3.15), vacuum formation (if any) is expected to be initiated at the origin (see section 6). For short time intervals one would therefore expect that the outer boundary condition does not significantly influence the behavior of the solution near the origin.

4. The isentropic Euler equations. The isentropic Euler equations are easily obtained from (3.13), (3.14) by formally taking the limit $\operatorname{Re} \rightarrow \infty$, i.e.,

$$
\begin{aligned}
\rho_{t}+(\rho u)_{\xi} & =0, \\
\rho\left(u_{t}+u u_{r}\right)+\frac{1}{\gamma \mathrm{M}^{2}}\left(\rho^{\gamma}\right)_{r} & =0 .
\end{aligned}
$$

Copyright (C) by SIAM. Unauthorized reproduction of this article is prohibited. 
4.1. The 1D case. Let us consider (4.1), (4.2) together with the Riemann data

$$
\rho(r, 0)=1 \quad \text { for all } r, \quad u(r, 0)=\left\{\begin{aligned}
-1 & \text { if } r<0 \\
1 & \text { if } r>0
\end{aligned}\right.
$$

As is well known, the above Riemann problem can easily be solved. Interestingly, the isothermal case, $\gamma=1$, and the general isentropic case, $\gamma>1$, are quite different.

The isothermal case $\gamma=\mathbf{1}$. For the "symmetric data" (4.3), the solution is found to consist of two rarefaction waves:

$$
\left[\begin{array}{l}
\rho \\
u
\end{array}\right](r, t)=\left\{\begin{array}{cl}
{\left[\begin{array}{c}
1 \\
-1
\end{array}\right]} & \text { if } \frac{r}{t}<-1-\frac{1}{\mathrm{M}}, \\
{\left[\begin{array}{c}
e^{-\left(\mathrm{M} \frac{r}{t}+\mathrm{M}+1\right)} \\
\frac{r}{t}+\frac{1}{\mathrm{M}}
\end{array}\right]} & \text { if }-1-\frac{1}{\mathrm{M}}<\frac{r}{t}<-\frac{1}{\mathrm{M}}, \\
{\left[\begin{array}{c}
e^{-\mathrm{M}} \\
0
\end{array}\right]} & \text { if }-\frac{1}{\mathrm{M}}<\frac{r}{t}<\frac{1}{\mathrm{M}}, \\
{\left[\begin{array}{c}
e^{\mathrm{M} \frac{r}{t}-\mathrm{M}-1} \\
\frac{r}{t}-\frac{1}{\mathrm{M}}
\end{array}\right]} & \text { if } \frac{1}{\mathrm{M}}<\frac{r}{t}<1+\frac{1}{\mathrm{M}}, \\
{\left[\begin{array}{l}
1 \\
1
\end{array}\right]} & \text { if } 1+\frac{1}{\mathrm{M}}<\frac{r}{t} .
\end{array}\right.
$$

Note that, regardless of the values of the Mach number, M, the above solution does not lead to cavitation. Indeed, the smallest value of the density is found to be $e^{-\mathrm{M}}$.

The case $\gamma>1$. Again, the solution is found to consist of two rarefaction waves for the data (4.3). However, in the present case, cavitation can occur. More precisely, if $M>\frac{2}{\gamma-1}$, the solution $\left[\begin{array}{l}\rho \\ u\end{array}\right](r, t)$ is given by

$$
\left\{\begin{array}{cl}
{\left[\begin{array}{c}
1 \\
-1
\end{array}\right]} & \text { if } \frac{r}{t}<-1-\frac{1}{\mathrm{M}}, \\
{\left[\begin{array}{c}
\left(\frac{2}{\gamma+1}-\mathrm{M} \frac{\gamma-1}{\gamma+1}\left(1+\frac{r}{t}\right)\right)^{2 /(\gamma-1)} \\
\frac{1}{\mathrm{M}(\gamma+1)}\left(2+(1-\gamma) \mathrm{M}+2 \mathrm{M} \frac{r}{t}\right)
\end{array}\right]} & \text { if }-1-\frac{1}{\mathrm{M}}<\frac{r}{t}<-1+\frac{2}{\gamma-1} \frac{1}{\mathrm{M}} \\
{\left[\begin{array}{l}
0 \\
\emptyset
\end{array}\right]} & \text { if }-1+\frac{2}{\gamma-1} \frac{1}{\mathrm{M}}<\frac{r}{t}<1-\frac{2}{\gamma-1} \frac{1}{\mathrm{M}} \\
{\left[\begin{array}{c}
\left(\frac{2}{\gamma+1}+\mathrm{M} \frac{\gamma-1}{\gamma+1}\left(-1+\frac{r}{t}\right)\right)^{2 /(\gamma-1)} \\
\frac{1}{\mathrm{M}(\gamma+1)}\left(-2+(-1+\gamma) \mathrm{M}+2 \mathrm{M} \frac{r}{t}\right)
\end{array}\right]} & \text { if } 1-\frac{2}{\gamma-1} \frac{1}{\mathrm{M}}<\frac{r}{t}<1+\frac{1}{\mathrm{M}} \\
{\left[\begin{array}{l}
1 \\
1
\end{array}\right]} & \text { if } 1+\frac{1}{\mathrm{M}}<\frac{r}{t} .
\end{array}\right.
$$

Note that, for this latter solution, no velocity $u$ is specified in the vacuum. If, on the other hand, the fluid is not sheared as hard, i.e., if $0<\mathrm{M}<\frac{2}{\gamma-1}$, then no cavitation 
takes place and the solution $\left[\begin{array}{l}\rho \\ u\end{array}\right](r, t)$ is found to be

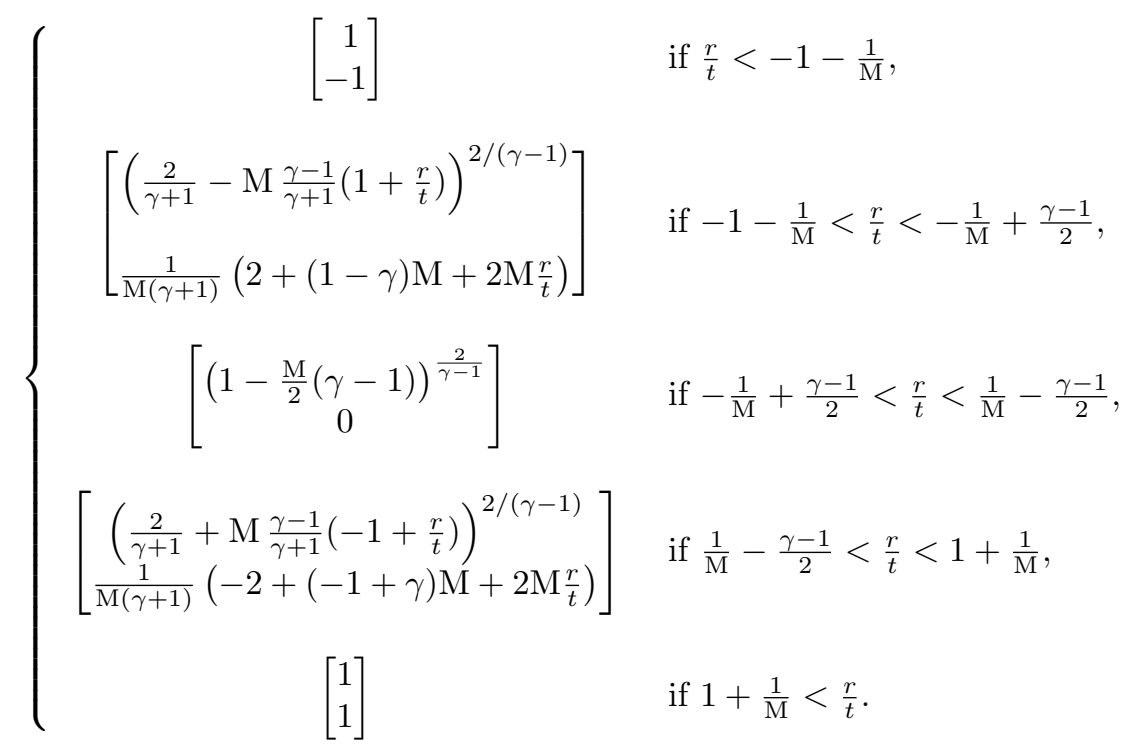

4.2. The multidimensional axisymmetric case. Similarity solutions to the Euler equations have also been considered for the 2D and 3D axisymmetric problems that are studied in this paper. Even though no closed form solutions can be found, the analysis reveals that solutions without swirls may exhibit cavitation if $\gamma>1$ but not if $\gamma=1$; see [43, section 7.4].

More precisely, let us concentrate on the case $\gamma>1$. Following [43], self-similar solutions to (4.1), (4.2) are sought in the form

$$
\rho=\rho(s), \quad u=u(s), \quad \text { where } s=\frac{t}{r} .
$$

This leads to the ODE system

$$
\begin{aligned}
\rho_{s} & =m \frac{\rho u(1-s u)}{s^{2} c^{2}-(1-s u)^{2}}, \\
u_{s} & =m \frac{s c^{2} u}{s^{2} c^{2}-(1-s u)^{2}}, \\
\rho(0) & =1, \quad u(0)=1,
\end{aligned}
$$

where $c=\frac{1}{M} \rho^{\frac{\gamma-1}{2}}$. The generalization of the $2 \mathrm{D}$ results of [43] to $3 \mathrm{D}$ problems is straightforward. It is presented here for the sake of completeness. Introducing the variables

$$
I=s u \quad \text { and } \quad K=s c,
$$

relations (4.4), (4.5) can be written

$$
\begin{aligned}
\frac{d I}{d \tau} & =I\left((1-I)^{2}-(1+m) K^{2}\right) \equiv I \mathcal{F}(I, K), \\
\frac{d K}{d \tau} & =K\left((1-I)^{2}-K^{2}-\frac{m}{2}(\gamma-1) I(1-I)\right) \equiv K \mathcal{G}(I, K), \\
\frac{d s}{d \tau} & =s\left((1-I)^{2}-K^{2}\right),
\end{aligned}
$$

Copyright (c) by SIAM. Unauthorized reproduction of this article is prohibited. 

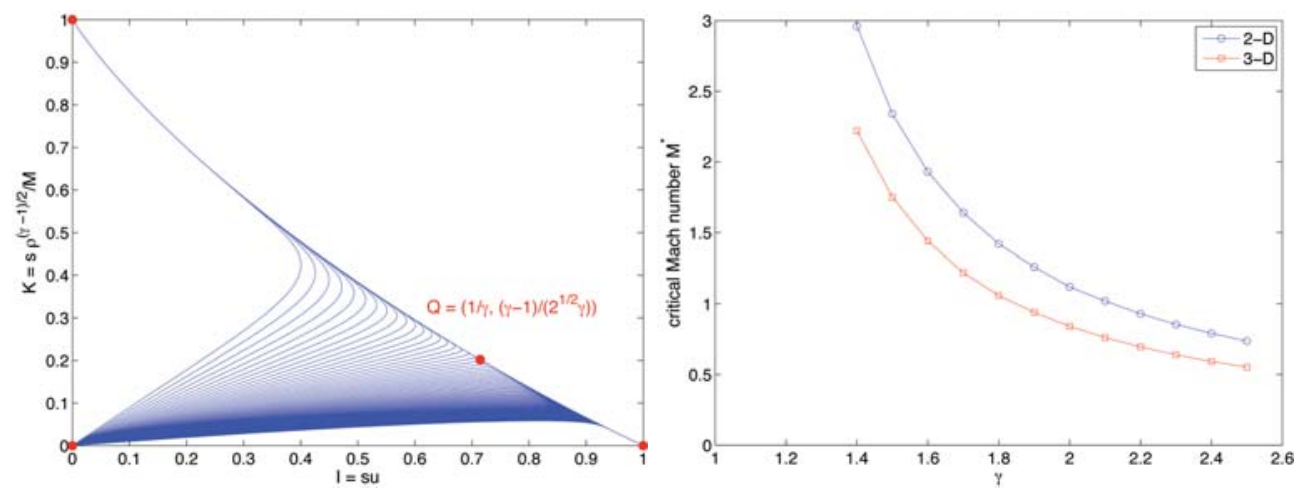

Fig. 4.1. Left: Phase diagram $(I=s u, K=s c)$ of the solutions to (4.7), (4.8), (4.9) for $m=1$ (2D) and $\gamma=1.4$. Solutions "leaving" $(0,0)$ are represented for values of the Mach number $M=1.1,1.2,1.3, \ldots, 10$. Right: Dependency of the critical Mach number $M^{\star}$ on $\gamma$ for $2 D$ and $3 D$ problems as obtained through numerical integration of (4.4), (4.5), (4.6). Vacuum takes place if and only if $M>M^{\star}$.

with the obvious definitions for $\mathcal{F}$ and $\mathcal{G}$ and where $\tau$ is a new independent variable defined by (4.9), which is introduced to make (4.7), (4.8) an autonomous system. In the $(I, K)$-phase plane, the stationary points of $(4.7),(4.8)$ are found to be

$$
(0,0),(1,0),(0,1) \quad \text { and } \quad Q=\left(\frac{2}{\gamma(1+m)+1-m}, \frac{1}{\sqrt{1+m}} \frac{\gamma(1+m)-1-m}{\gamma(1+m)+1-m}\right) .
$$

Let us consider the region $\Omega \subset \mathbb{R}^{2}$ defined by

$$
\begin{aligned}
\Omega=\{(I, K) & ;>0, K>0, \\
\mathcal{G} & >0 \text { for } 0<I \leq \frac{2}{\gamma(1+m)+1-m}, \\
\mathcal{F} & \left.>0 \text { for } \frac{2}{\gamma(1+m)+1-m} \leq I<1\right\} .
\end{aligned}
$$

A simple sign study along $\partial \Omega$ shows that $\Omega$ is invariant under (4.7), (4.8). The system (4.7), (4.8), (4.9) then has integral curves from $(0,0)$ to either $(0,1),(1,0)$ or $Q$.

Lemma 1. Let $m=1$ (two space dimensions) or 2 (three space dimensions) and let $\gamma>1$. Then there exists $M^{\star}=M^{\star}(m, \gamma)>0$ such that

- if $M<M^{\star}$, the solution ( $\left.\rho, u\right)$ of (4.4), (4.5), (4.6) is defined for $0<s<\bar{s}<$ $\infty$, where $\bar{s}$ is such that $(\rho(\bar{s}), u(\bar{s}))=(\bar{\rho}, 0)$ (convergence to $(I, K)=(0,1)$ at a finite s-value, no vacuum);

- if $M>M^{\star}$, the solution ( $\left.\rho, u\right)$ of (4.4), (4.5), (4.6) is defined for $0<s<\bar{s}<$ $\infty$, where $\bar{s}$ is such that $(\rho(\bar{s}), u(\bar{s}))=(0, \bar{u})$ (convergence to $(I, K)=(1,0)$ at a finite s-value, vacuum);

- if $M=M^{\star}$, the solution $(\rho, u)$ of (4.4), (4.5), (4.6) is defined for $0<s<\infty$ and converges to $(I, K)=Q$ as $s \rightarrow \infty$ (critical case).

Proof. The proof follows in a straightforward way from [43, section 7.4]

There does not appear to be an explicit formula for the critical value $M^{\star}$. However, equations (4.4), (4.5), (4.6) can be solved numerically and an approximate value of $M^{\star}$ inferred from the corresponding results. The feasibility of this approach is illustrated in Figure 4.1(left), which is in exact agreement with the above lemma. 
The above observations provide a relatively easy way to numerically investigate vacuum formation for the multidimensional Euler equations. A standard ODE solver can be used to integrate the above equations. It is worth noting that the points $(I, K)=(0,1)$ or $(1,0)$ are reached for finite values of $s$; the solution can then be continuously extended toward $r=0$. We omit the details. Figure 4.1(right) illustrates the dependency of $M^{\star}$ on $\gamma$ for 2D and 3D problems as found through numerical investigation.

5. Discretization and numerical analysis. The numerical approach for solving the Navier-Stokes equations (3.9), (3.10), (3.11) under the specific assumptions considered here is based on a splitting between the Euler equations, on the one hand, and a diffusive equation, on the other hand. This way, one can take advantage of the similarity solutions considered in the previous section (which can be solved to a high degree of accuracy by ODE solvers; see below). At each space/time node, such a solution is locally constructed. This process is akin to numerically solving families of local Riemann problems, as is routinely done in many numerical schemes for hyperbolic conservation laws; see, e.g., [27].

The splitting algorithm is illustrated in the case of the barotropic equations (4.1), (4.2). Let $\left(\rho_{n}, u_{n}\right)$ be the solution at some time $t_{n}=n \Delta t$, where $\Delta t$ is the time step. To obtain the solution $\left(\rho_{n+1}, u_{n+1}\right)$ at a later time $t_{n+1}=(n+1) \Delta t$, an "Euler step" is first taken; i.e., one solves $(4.1),(4.2)$ from $t_{n}$ to $t_{n+1}$ with the initial condition

$$
\rho\left(\cdot, t_{n}\right)=\rho_{n}, \quad u\left(\cdot, t_{n}\right)=u_{n} .
$$

The resulting solution at time $t_{n+1}$ is denoted $\left(\rho^{\star}, u^{\star}\right)$. The following diffusive step is then taken:

$$
\begin{aligned}
\rho^{\star} u_{t} & =\frac{1}{\operatorname{Re}} u_{\xi r}, \quad t \in\left(t_{n}, t_{n+1}\right), \\
u\left(\cdot, t_{n}\right) & =u^{\star} .
\end{aligned}
$$

As both steps have to be solved numerically, their respective discretization is now described. For notational convenience, the diffusive step is described first.

5.1. The diffusive step. Equation (5.1) is discretized in space using Chebyshev collocation methods [3]. Such methods deliver high accuracy with a low number of nodes for smooth solutions (which are expected here for $t>0$ ). To circumvent the coordinate singularity at $r=0$ of the 2D and 3D problems, Chebyshev-Gauss-Radau nodes are used instead of the more common Chebyshev-Gauss-Lobatto nodes. In the spatial domain $(0,1)$ those nodes have location

$$
r_{j}=\frac{1}{2}\left(1+\cos \left(\frac{2 \pi j}{2 N-1}\right)\right), \quad j=0, \ldots, N-1 .
$$

In each case, $N$ stands for the number of nodes. For $r \in(0,1)$ and $t>0$, we seek an approximation $u_{N}$ of $u$ of the form

$$
u_{N}(r, t)=\sum_{i=0}^{N-1} U_{i}(t) \psi_{i}(r),
$$

where $\left\{\psi_{i}\right\}_{i=0}^{N-1}$ are the Lagrange interpolation polynomials at the ChebyshevGauss-Lobatto/Radau nodes on $[0,1]$, i.e., $\psi_{i}\left(x_{j}\right)=\delta_{i j}$. 
Interpolation at one of the above sets of nodes of a function $v=v(r, t)$ simply takes the form

$$
I_{N} v(r, t)=\sum_{j=0}^{N-1} v\left(r_{j}, t\right) \psi_{j}(r) .
$$

By definition, the Chebyshev collocation derivative of $v$ with respect to $r$ at those nodes is then

$$
\frac{\partial}{\partial r}\left(I_{N} v\right)\left(r_{l}, t\right)=\sum_{j=0}^{N-1} v\left(r_{j}, t\right) \psi_{j}^{\prime}\left(r_{l}\right)=\sum_{j=0}^{N-1} D_{l j} v\left(r_{j}, t\right),
$$

with $D_{l j}=\psi_{j}^{\prime}\left(r_{l}\right)$. The collocation derivative at the nodes can then be obtained through matrix multiplication.

The discrete velocity $u_{N}$ takes the form

$$
u_{N}(r, t)=\sum_{i=0}^{N-1} U_{i}(t) \psi_{i}(r) .
$$

The semidiscretized in space problem (5.1) has the form

$$
Z_{U}\left(\left(I_{N} \rho^{\star}\right) \cdot * U^{\prime}\right)=\left(D^{2}+m \operatorname{diag}(1 . / R) D-m \operatorname{diag} 1 . /\left(R .{ }^{\wedge} 2\right)\right) U+B_{U},
$$

with the obvious notation for $U$ and $D$; the vector $R$ is the node vector, $R_{j}=r_{j}$, $j=0, \ldots, N-1$. The matrix $Z_{U}$ zeroes the first and/or the last entry(ies) of a vector, and further $B_{U}$ is a vector related to the boundary conditions (to be specified below). In the above equations, a "dotted operation" (for instance .*) refers to that operation being performed elementwise (for instance, $U . * V$ is the vector of $i$ th component $U_{i} V_{i}$, $i=0, \ldots, N-1)$.

The computations are carried out with the boundary condition (3.16). In case the density vanishes (or becomes very small) at some node $r_{i}$ at time $t$, i.e., $\mathcal{I}_{N} \rho^{\star}\left(r_{i}, t\right)=0$, then the differential equation for $\mathcal{U}_{i}(t)$ degenerates into an algebraic equation; in other words, (5.3) becomes differential algebraic. How much of a numerical problem this is depends on the index of the system. The minimum number of times one has to differentiate all or part of (5.3) to recover an ODE system is the index of the differential algebraic equation (DAE) [1]. Here the index is easily found to be equal to 1. Indeed, differentiating the algebraic equation for $\mathcal{U}_{i}$ leads to an ODE, provided that the operator $D^{2}+m \operatorname{diag}(1 . / R) D-m \operatorname{diag} 1 . /\left(R .{ }^{\wedge} 2\right)$ with proper side conditions applied to $U^{\prime}$ is nonsingular. The side conditions are that the velocity is fixed on the outer boundary and thus $U_{1}^{\prime}(t)=0$ and that nonsingular solutions are sought. The above operator can be checked to be nonsingular by direct inspection of the matrices involved. Alternatively, at the continuous level, one can check that the only nonsingular solution to $\partial_{r r} \dot{u}+m / r \partial_{r} \dot{u}-m \dot{u} / r^{2}=0$ with $\dot{u}(1)=0$ is the trivial solution $\dot{u} \equiv 0$ (where $\dot{u}=u_{t}$ ).

In the presence of vacuum, the system (5.3) is a DAE that is semiexplicit of index 1; as such it is amenable to relatively simple time discretization such as BDF [1]. Here, we used the MATLAB routine ODE15s, which implements a variant of BDF [35].

5.2. The Euler step. Let $\left(\rho_{n}\left(r_{j}\right), u_{n}\left(r_{j}\right)\right)$ be given values for the density and velocity at time $t^{n}$ at the node $r_{j}, j=0, \ldots, N-1$. A family of $N$ similarity solutions 


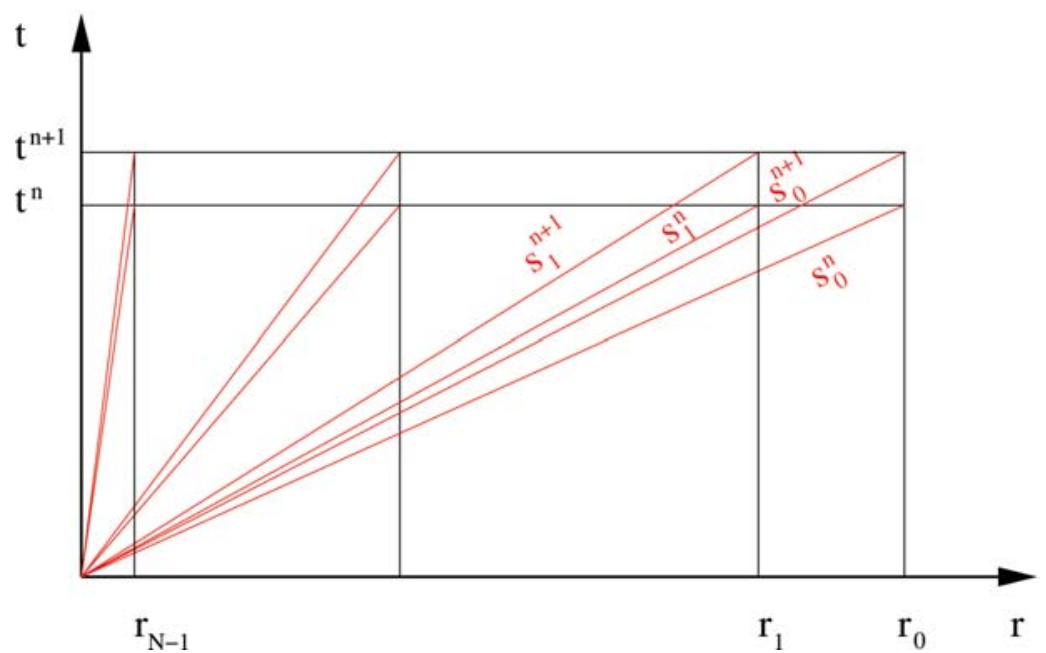

FiG. 5.1. Meshes used for the diffusive step $\left(\left\{r_{j}, t^{n}\right\}_{j=0, n=0}^{N-1, N_{t}}\right)$ and Euler step $\left(\left\{s_{j}^{n}\right\}_{j=0, n=0}^{N-1, N_{t}}\right)$.

is now defined in the same way as in section 4.1. More precisely, let $s_{j}^{n}=\frac{t^{n}}{r_{j}}$ be the slopes corresponding to the above data points, and let $s_{j}^{n+1}=\frac{t^{n+1}}{r_{j}}$ be the slopes at the next time step; see Figure 5.1. Then, for each $j=0, \ldots, N-1$, equations (4.4), (4.5) are solved from $s=s_{j}^{n}$ to $s_{j}^{n+1}$ with initial conditions

$$
\rho\left(s_{j}^{n}\right)=\rho_{n}\left(r_{j}\right), \quad u\left(s_{j}^{n}\right)=u_{n}\left(r_{j}\right) .
$$

5.3. The splitting algorithm. For given Mach and Reynolds numbers, and given spatial and temporal resolutions, i.e., $N$ and $\Delta t$ being chosen, the following steps are taken for $N_{t}$ time steps:

- initialize $\rho_{0}$ and $u_{0}$ according to (3.15),

- for $n=0$ to $N_{t}-1$

- for $j=N-1$ to 0 by -1

* EULER: solve (4.4), (4.5), (5.4)

* set $\rho^{\star}\left(r_{j}\right)=\rho\left(s_{j}^{n+1}\right)=\rho_{n+1}\left(r_{j}\right)$ and $u^{\star}\left(r_{j}\right)=u\left(s_{j}^{n+1}\right)$

- end

- DIFFUSION: solve (5.1), (5.2) through (5.3)

- set $u_{n+1}\left(r_{j}\right)=u\left(r_{j}, t_{n+1}\right), j=0, \ldots, N-1$

- end

6. Numerical results. The problem (3.13), (3.14) has been solved using the method described in the previous section with various values of the physical parameters (Mach number M, Reynolds number Re, and adiabatic coefficient $\gamma$ ). The initial and boundary conditions are given by (3.15) and (3.16), respectively. The mesh size is fixed at $N=32$ for all the results given below.

For all our examples, the numerical density $\rho_{N}$ is an increasing function of $r$, and thus the numerical solution is probed at the node closest to the origin for vacuum detection. Vacuum formation, if it occurs, is expected to take place during a fast initial transient phase. A comparison with the inviscid case is instructive. If cavitation takes place for the Euler equations, it does so instantaneously (at the origin), i.e., for $t=0+$. For the $1 \mathrm{D}$ case, this fact is obvious from the explicit self-similar solutions given in 

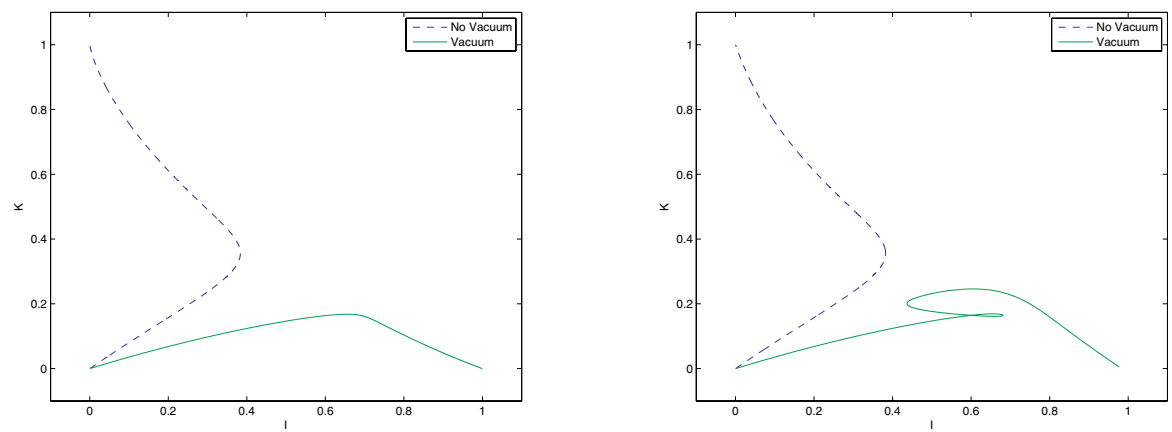

FIG. 6.1. Phase diagram corresponding to the evolution of the solutions, at the node closest to the origin, of the Euler solution (left) and the Navier-Stokes solution (right) (see section 4 for definition of $I$ and $K)$. The parameters are taken as $m=2(3 D)$ and $\mathrm{M}=1.2$ (no vacuum) and $\mathrm{M}=2.7$ (vacuum); for the Navier-Stokes solution (right), the Reynolds number Re is $10^{6}$.

section 4. Regarding the Navier-Stokes equations, our efforts are concentrated on the multidimensional cases for which there is no node at the origin. For the node closest to the origin, phase space trajectories can be used in a way similar to what was done in Figure 4.1(left), giving a clear picture of the evolution of the density there. Using the same notation as in section 4, Figure 6.1 illustrates the difference between the evolution of the Euler solutions (left panel) and Navier-Stokes solutions (right panel).

Based on the above remarks, a specific calculation is said to lead to vacuum formation if for some time $t, 0<t<.005$,

$$
\rho_{N}\left(r_{N-1}, t\right)<t o l=10^{-14}
$$

where $r_{N-1}=\frac{1}{2}\left(1+\cos \left(\frac{2 N-1}{2 N-2} \pi\right)\right)$ is the Chebyshev-Gauss-Radau node closest to the origin. In some cases, the time asymptotic behavior of the solution at $r_{N-1}$ was not clear based on a phase plane analysis. Those cases are reported below as inconclusive, even if the density itself satisfied (6.1).

6.1. The $1 \mathrm{D}$ case. The above method cannot be used directly in the $1 \mathrm{D}$ case. Using an adapted method (details are omitted), no vacuum formation was numerically observed for the 1D Navier-Stokes solution as is shown in Figure 6.2. The absence of vacuum in the solutions to the $1 \mathrm{D}$ Navier-Stokes system is consistent with the solutions found by Hoff [15], who considers discontinuous data of the same type as in the present paper.

6.2. The multi-D barotropic case. The $2 \mathrm{D}$ and $3 \mathrm{D}$ barotropic flows (3.13), (3.14) with initial and boundary conditions (3.15), (3.16) are solved, for fixed values of the adiabatic coefficient $\gamma$, on grids in "Reynolds and Mach number space," i.e., for a collection of values of those two parameters.

Figure 6.3 corresponds to the value $\gamma=1.4$ for the $2 \mathrm{D}$ and $3 \mathrm{D}$ cases, in the left and right panels, respectively. As explained above, the gray area corresponds to values of the parameters for which the numerical calculations were inconclusive, for instance due to the absence of a clear asymptotic behavior in phase space during the allotted computational time. It clearly illustrates that for large enough values of $\mathrm{M}$ there appears to be vacuum formation. The displayed results are relatively insensitive to 

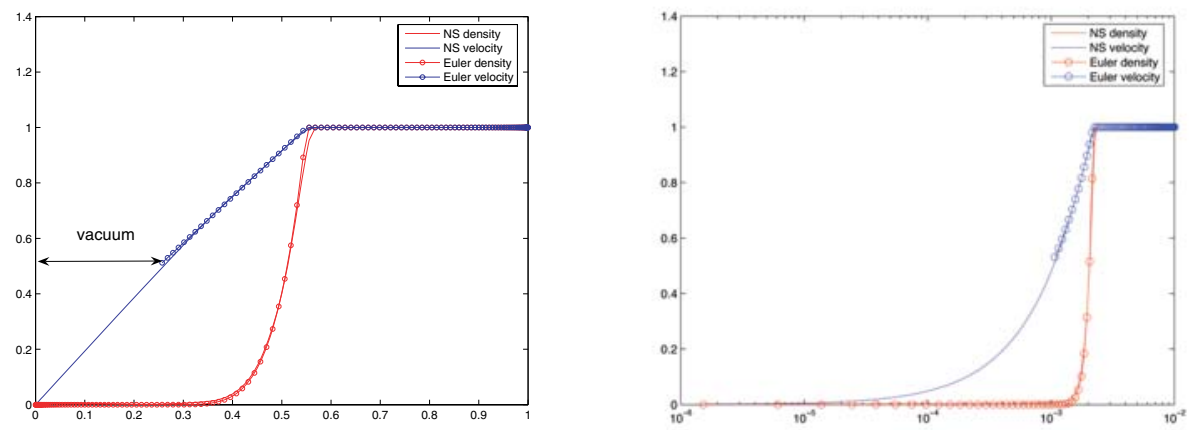

FIG. 6.2. $1 D$ Navier-Stokes and Euler solutions for $\gamma=1.4, \mathrm{M}=10$. Left: Solutions at time $t=0.5$ with $\operatorname{Re}=10,000$ (for the Navier-Stokes flow). Right: Solutions at time $t=.002$ with $\operatorname{Re}=1,000,000$ (for the Navier-Stokes flow). For these values, both the $1 D$ Euler solution and the multi-D Navier-Stokes solution exhibit cavitation (see Figure 6.3 below), while the $1 D$ Navier-Stokes solution does not.
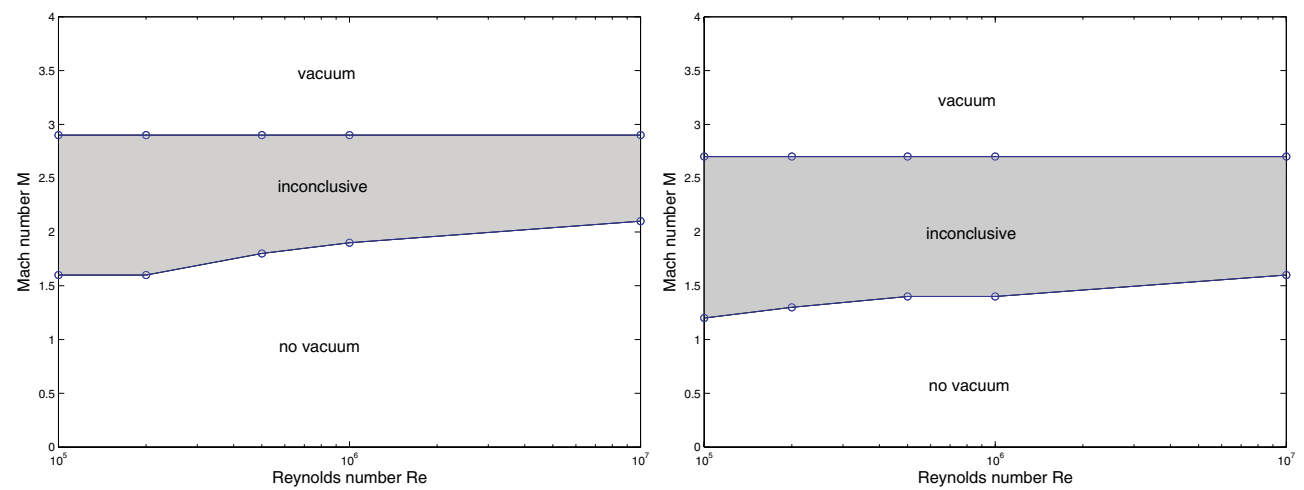

FIG. 6.3. Vacuum formation for $2 D$ (left) and $3 D$ (right) barotropic flows (3.13), (3.14) with initial and boundary conditions (3.15), (3.16) and $\gamma=1.4$.

the discretization parameters. It is also observed that vacuum is more easily formed in three than in two dimensions, in agreement with the remarks in section 2.1.2.

Similar results were observed for larger values of $\gamma$ : it gets easier to create vacuum as $\gamma$ increases.

6.3. The full system. As mentioned in section 4.2 , the construction of selfsimilar solutions for the nonisentropic Euler equations (3.1)-(3.3) appears to be open. Therefore, we do not have the benefit of using this tool as part of the numerical algorithm. While more research is needed, preliminary calculations based on an unsplit algorithm indicate vacuum formation here as well.

7. Conclusion. Our numerical results indicate that vacuum formation is possible in solutions of the multidimensional compressible Navier-Stokes equations. This applies to discontinuous and sufficiently large data where the initial density is uniformly bounded away from zero. The same numerical code gives results for one-dimensional flow that are in agreement with the known analytical results. The conclusions do not contradict the currently known results for multi-D flow. 
No attempt was made to follow the solutions past vacuum formation. The present study also leaves unanswered the issue of whether vacuum formation is instantaneous, as is the case for the corresponding solutions to the Euler equations.

Acknowledgments. The last author is indebted to David Hoff for several discussions about the vacuum problem in compressible flow. The clarity and accuracy of this article were significantly improved by the criticism of an anonymous referee.

\section{REFERENCES}

[1] K. E. Brenan, S. L. Campbell, and L. R. Petzold, Numerical Solution of Initial-Value Problems in Differential-Algebraic Equations, Classics in Applied Mathematics 14, SIAM, Philadelphia, 1995.

[2] D. Bresch And B. Desjardins, Stabilité de solutions faibles globales pour les équations de Navier-Stokes compressible avec température [Stability of global weak solutions for the Navier-Stokes equations modelling compressible and heat-conducting fluids], C. R. Math. Acad. Sci. Paris, 343 (2006), pp. 219-224 (in French).

[3] C. Canuto, M. Y. Hussaini, A. Quarteroni, and T. A. Zang, Spectral Methods in Fluid Dynamics, Springer Ser. Comput. Dynam., Springer, Berlin, 1987.

[4] C. M. DAfermos, Global smooth solutions to the initial-boundary value problem for the equations of one-dimensional nonlinear thermoviscoelasticity, SIAM J. Math. Anal., 13 (1982), pp. 397-408.

[5] C. M. Dafermos and L. Hsiao, Global smooth thermomechanical processes in one-dimensional nonlinear thermoviscoelasticity, Nonlinear Anal., 6 (1982), pp. 435-454.

[6] R. Danchin, Global existence in critical spaces for compressible Navier-Stokes equations, Invent. Math., 141 (2000), pp. 579-614.

[7] R. Danchin, Global existence in critical spaces for compressible viscous and heat conductive gases, Arch. Ration. Mech. Anal., 160 (2001), pp. 1-39.

[8] R. DANCHIN, On the uniqueness in critical spaces for compressible Navier-Stokes equations, NoDEA, Nonlinear Differential Equations Appl., 12 (2005), pp. 111-128.

[9] R. DuAn AND Y. ZhaO, A note on the non-formation of vacuum states for compressible NavierStokes equations, J. Math. Anal. Appl., 311 (2005), pp. 744-754.

[10] E. Feireisl, A. Novotný, And H. Petzeltová, On the existence of globally defined weak solutions to the Navier-Stokes equations, J. Math. Fluid Mech., 3 (2001), pp. 358-392.

[11] E. FeIreisl, Dynamics of Viscous Compressible Fluids, Oxford University Press, London, 2004.

[12] E. Hairer and G. Wanner, Solving Ordinary Differential Equations II: Stiff and Differential Algebraic Problems, Springer Ser. Comput. Math. 14, Springer, Berlin, 1996.

[13] D. HofF, Spherically symmetric solutions of the Navier-Stokes equations for compressible, isothermal flow with large, discontinuous initial data, Indiana Univ. Math. J., 41 (1992), pp. 1225-1302.

[14] D. Hoff, Discontinuous solutions of the Navier-Stokes equations for multidimensional flows of heat-conducting fluids, Arch. Ration. Mech. Anal., 139 (1997), pp. 303-354.

[15] D. HofF, Global solutions of the equations of one-dimensional, compressible flow with large data and forces, and with differing end states, Z. Angew. Math. Phys., 49 (1998), pp. $774-785$.

[16] D. HofF, Uniqueness of weak solutions of the Navier-Stokes equations of multidimensional, compressible flow, SIAM J. Math. Anal., 37 (2006), pp. 1742-1760.

[17] D. Hoff And H. K. Jenssen, Symmetric nonbarotropic flows with large data and forces, Arch. Ration. Mech. Anal., 173 (2004), pp. 297-343.

[18] D. Hoff ANd D. Serre, The failure of continuous dependence on initial data for the NavierStokes equations of compressible flow, SIAM J. Appl. Math., 51 (1991), pp. 887-898.

[19] D. Hoff And J. Smoller, Non-formation of vacuum states for compressible Navier-Stokes equations, Comm. Math. Phys., 216 (2001), pp. 255-276.

[20] S. JiAng AND P. Zhang, Global weak solutions to the Navier-Stokes equations for a $1 D$ viscous polytropic ideal gas, Quart. Appl. Math., 61 (2003), pp. 435-449.

[21] YA. I. KANEL, A model system of equations for the one-dimensional motion of a gas, Differ. Uravn., 4 (1968), pp. 721-734.

[22] S. Kawashima, Systems of Hyperbolic-Parabolic Composite Type, with Applications to the Equations of Magnetohydrodynamics, Ph.D. thesis, Department of Mathematics, Kyoto University, Kyoto, Japan, 1983. 
[23] S. KaWAShima And T. Nishida, Initial-boundary value problems for the equations of motion of compressible viscous and heat-conductive fluids, Comm. Math. Phys., 89 (1983), pp. 445-464.

[24] B. KAwOHL, Global existence of large solutions to initial boundary value problems for a viscous, heat-conducting, one-dimensional real gas, J. Differential Equations, 58 (1985), pp. 76-103.

[25] A. V. Kazhikhov, Sur la solubilité globale des problèmes monodimensionnels aux valeurs initiales-limitées pour les équations d'un gaz visqueux et calorifère, C. R. Acad. Sci. Paris, Sér. A, 284 (1977), pp. 317-320 (in French).

[26] A. V. Kazhikhov and V. V. Shelukhin, Unique global solution with respect to time of initialboundary value problems for one-dimensional equations of a viscous gas, J. Appl. Math. Mech., 41 (1977), pp. 273-282; translated from Prikl. Mat. Meh., 41 (1977), pp. 282-291 (in Russian).

[27] R. J. Leveque, Finite Volume Methods for Hyperbolic Problems, Cambridge University Press, Cambridge, UK, 2002.

[28] P. L. Lions, Mathematical Topics in Fluid Mechanics, Vol. 2, Compressible Models, Oxford University Press, London, 1998.

[29] T. P. Liu, Z. Xin, And T. YAng, Vacuum states for compressible flow, Discrete Contin. Dynam. Systems, 4 (1998), pp. 1-32.

[30] T. LuO, Z. Xin, AND T. YANG, Interface behavior of compressible Navier-Stokes equations with vacuum, SIAM J. Math. Anal., 31 (2000), pp. 1175-1191.

[31] A. Matsumura and T. Nishida, The initial value problem for the equations of motion of viscous and heat-conductive gases, J. Math. Kyoto U., 20 (1980), pp. 67-104.

[32] A. Mellet And A. Vasseur, Existence and Uniqueness of Global Strong Solutions for OneDimensional Compressible Navier-Stokes Equations, preprint, Mathematics Department, University of Texas at Austin, 2006.

[33] A. Novotný and I. Stras̆Kraba, Introduction to the Mathematical Theory of Compressible Flow, Oxford University Press, London, 2004.

[34] J. SERRIN, Mathematical principles of classical fluid mechanics, in Handbuch der Physik, Bd. 8/1, Strömungsmechanik I, Springer-Verlag, Berlin, 1959, pp. 125-263.

[35] L. F. Shampine, M. W. Reichelt, and J. A. Kierzenka, Solving index-1 DAEs in MATLAB and Simulink, SIAM Rev., 41 (1999), pp. 538-552.

[36] J. Smoller, Shock Waves and Reaction-Diffusion Equations, 2nd ed., Grundlehren Math. Wiss. 258, Springer-Verlag, New York, 1994.

[37] Z. XIN, Blowup of smooth solutions to the compressible Navier-Stokes equation with compact density, Comm. Pure Appl. Math., 51 (1998), pp. 229-240.

[38] Z. XIN AND H. YuAn, Vacuum state for spherically symmetric solutions of the compressible Navier-Stokes equations, J. Hyperbolic Differential Equations, 3 (2006), pp. 403-442.

[39] T. YANG, Z. YAO, AND C. ZHU, Compressible Navier-Stokes equations with density-dependent viscosity and vacuum, Comm. Partial Differential Equations, 26 (2001), pp. 965-981.

[40] T. YANG AND H. ZHAO, A vacuum problem for the one-dimensional compressible NavierStokes equations with density-dependent viscosity, J. Differential Equations, 184 (2002), pp. 163-184.

[41] T. YAng AND C. ZHU, Compressible Navier-Stokes equations with degenerate viscosity coeffcient and vacuum, Comm. Math. Phys., 230 (2002), pp. 329-363.

[42] S.-W. Vong, T. YAng, AND C. ZHU, Compressible Navier-Stokes equations with degenerate viscosity coefficient and vacuum. II, J. Differential Equations, 192 (2003), pp. 475-501.

[43] Y. Zheng, Systems of Conservation Laws: Two-Dimensional Riemann Problems, Birkhäuser Boston, Cambridge, MA, 2001.

Copyright $@$ by SIAM. Unauthorized reproduction of this article is prohibited. 